Revista PSICOLOGIA, 2020, Vol. 34 (1), 82-93. doi: 10.17575/psicologia.v34i1.1474

\title{
0 benefício do enriquecimento trabalho-família para a saúde dos professores: 0 papel da autoeficácia percebida num estudo diário
}

\author{
Leonor Milheiro de Vilhena ${ }^{1} \&$ Susana Tavares ${ }^{2}$ \\ ${ }^{1}$ Instituto Universitário de Lisboa, ISCTE-IUL \\ 2 Instituto Universitário de Lisboa, ISCTE-IUL, Business Research Unit - BRU (UNIDE-IUL)
}

\begin{abstract}
Resumo: 0 presente estudo pretendeu analisar de que forma o enriquecimento trabalho-família afecta a saúde dos professores numa base diária. Baseando-nos na teoria da conservação dos recursos (Hobfoll, 1989, 2002) hipotetizámos que o nível diário de enriquecimento trabalho-família (ETF) sentido aumentaria os recursos do indivíduo, nomeadamente a perceção que este tem da sua autoeficácia, o que, por sua vez, afetaria positivamente a sua saúde. Os 66 professores que participaram no estudo preencheram questionários durante 8 dias de trabalho consecutivos, resultando num total de 528 observações. Os resultados evidenciaram que nos dias em que os professores consideram que o seu trabalho enriquece o papel que desempenham na sua família, reportam um menor número de sintomas somáticos. Os resultados mostraram ainda que o efeito do ETF na saúde é parcialmente explicado pelo aumento da perceção de autoeficácia do indivíduo. As limitações do estudo, sugestões para pesquisas futuras, e implicações dos resultados são discutidas.
\end{abstract}

Palavras-chave: Enriquecimento trabalho-família; sintomas somáticos; perceção de autoeficácia; estudo diário.

The benefit of work-family enrichment for teachers' health: The role of perceived self-efficacy in a daily study. The present study aimed to analyze how work-family enrichment affects the health of teachers on a daily basis. Based on the conservation of resources theory (Hobfoll, 1989, 2002) we hypothesized that the daily level of work-family enrichment (WFE) would increase individual's resources, namely their selfefficacy which, in turn, would positively affect their health. The 66 teachers who participated in the study completed questionnaires during 8 consecutive days of work, resulting in a total of 528 observations. The results showed that on days when teachers consider that their work enriches the role they play in their family, they report fewer somatic symptoms. In addition, the results showed that WFE's effect on health is partially explained by the increase in the individual's perception of self-efficacy. The limitations of the study, as well as the theoretical and practical implications of the results found and the suggestions for future research, are discussed.

Keywords: Work-family enrichment; somatic symptoms; perceived self-efficacy; diary study.

O equilíbrio da vida profissional com a vida familiar é um desafio que se coloca tanto ao colaborador, como à organização em que este trabalha, no sentido de se assegurar uma harmonização saudável do seu envolvimento, quer no trabalho, quer na família. A literatura tem-se centrado na análise dos preditores e das consequências da interferência negativa do trabalho na família - conflito trabalho-família - perspetiva que assume que a participação em diversos papéis levará, em último caso, a um esgotamento dos recursos do indivíduo (e.g., Tavares \& Dias, 2014). Apenas mais recentemente, a atenção tem vindo a ser direcionada também para a influência positiva que o trabalho pode ter na vida familiar do colaborador. Este foco no enriquecimento trabalho-família (ETF) vem complementar a perspetiva centrada no conflito (Greenhaus \& Powell, 2006), permitindo assim ter uma visão mais equilibrada da interface trabalho-família. Apesar de na literatura alguns estudos terem analisado as consequências positivas do ETF para o bem-estar dos indivíduos (e.g., Carvalho \& Chambel, 2014, 2018; McNall, Nicklin, \& Masuda, 2010; McNall, Scott, \& Nicklin, 2015), poucos são os que se debruçaram sobre a relação do ETF com a saúde física do indivíduo (para exceções ver Carlson, Kacmar, Zivnuska, Ferguson, \& Whitten, 2011; Cho \& Chen, 2018; Russo, 2015; Sprung \& Jex, 2017). Acresce ainda que, com exceção de Russo (2015), os restantes autores se centraram

\footnotetext{
${ }^{1}$ Morada para correspondência: Susana M. Tavares, Departamento de Recursos Humanos e Comportamento Organizacional da ISCTE-IUL Business School, Instituto Universitário de Lisboa (ISCTE-IUL), Av. das Forças Armadas, Edifício ISCTE, 1649-026 Lisboa, Portugal. E-mail: susana.tavares@iscte-iul.pt. Este trabalho foi parcialmente apoiado no âmbito do projeto financiado pela Fundação para a Ciência e Tecnologia (FCT:UID/GES/00135/2013).
} 
na relação direta do ETF com o bem-estar ou com a saúde, omitindo o estudo dos mecanismos que podem explicar esta relação.

Assim, tendo por base a teoria da conservação dos recursos (TCR, Hobfoll, 1989, 2002), de acordo com a qual as pessoas tentam proteger e maximizar os seus recursos como forma de preservarem e mesmo aumentarem o seu bem-estar, o nosso estudo pretendeu analisar o impacto do ETF na saúde diária dos professores, nomeadamente nos sintomas somáticos por eles referidos diariamente. Pretendemos ainda estudar o papel da autoeficácia percebida para a explicação desta relação.

Optámos por analisar este fenómeno com professores, pois apesar de raramente, a interface trabalho-família ter sido estudada neste tipo de profissionais (para exceções ver Cinamon, 2009; Liu \& Cheng, 2015), as tarefas associadas a esta profissão - pesquisa actualizada de conhecimentos, gestão de conflitos em sala de aula, preparação de atividades extra-curriculares, etc. (Padrão, Pinto, \& Santos-Rita, 2012) - são potencialmente transferíveis para o contexto familiar, podendo contribuir para que o professor melhore o seu desempenho também neste contexto. Ainda, de acordo com De Simone, Cicotto e Lampis (2016), muitos estudos identificaram o ensino como sendo uma das profissões mais stressantes e os professores como tendo níveis mais baixos de saúde física e psicológica, quando comparados com outros grupos de profissionais (Kidger et al., 2016), nomeadamente por estarem sujeitos a ambientes de elevada exigência laboral e à necessidade de adaptação constante a alterações no sistema de ensino. Assim, tendo os professores um papel fulcral, não só para a motivação dos alunos para a aprendizagem, como para os resultados escolares por estes obtidos (Panayotis \& Kyrickides, 2013) e sendo um importante elo de ligação da escola à comunidade (Thoonen, Sleegers, Peestma, \& Oort, 2011), será importante analisar como podemos contribuir para que estes profissionais tenham níveis mais elevados de saúde.

\section{Enriquecimento trabalho-família e saúde}

O desenvolvimento de recursos é fundamental para o processo de enriquecimento trabalho-família. A perspectiva do ETF assume que o desempenho do papel no trabalho pode ter efeitos positivos para a vida familiar (Frone, 2003). Isto é, quando existe ETF os recursos gerados no domínio laboral possibilitam a melhoria da qualidade de vida familiar, quer pela via instrumental, quer pela via afetiva (Greenhaus \& Powell, 2006). Estes recursos podem dizer respeito a afeto, a oportunidades de desenvolvimento ou a capital (Carlson, Kacmar, Wayne, \& Grzywacz, 2006). Mais especificamente, Greenhaus e Powell (2006) referem que os recursos que facilitam o ETF podem ser físicos, psicológicos, relativos a competências, capacidades e ao acesso a diferentes perspectivas, recursos que propiciam a flexibilidade e ainda, recursos relativos a capital social ou a recursos materiais.

A perspetiva do ETF parte, então, da teoria da acumulação de papéis proposta por Sieber (1974) e da abordagem expansionista de Marks (1977). Estas defendem que a participação em diferentes papéis pode ser mais vantajosa para os indivíduos, podendo prevalecer sobre os efeitos negativos que as exigências dos vários papéis podem acarretar (Greenhaus \& Powell, 2006).

0 ETF apresenta-se ainda como um constructo bidirecional. Pode ocorrer na direção trabalhofamília, quando os recursos adquiridos no trabalho são aplicados com eficácia no domínio familiar; e pode ocorrer também na direção família-trabalho, quando os recursos obtidos no domínio familiar são aplicados com sucesso no domínio laboral. No presente estudo, focámo-nos especificamente na direção trabalhofamília, uma vez que as organizações mais facilmente poderão intervir no que se passa no local de trabalho do que no que se passa na família (Daniel \& Sonnentag, 2014).

A literatura tem evidenciado que a experiência de níveis mais elevados de ETF está associada a maior satisfação com o trabalho, com a família e com a vida (e.g., Aryee, Srinivas, \& Tan, 2005; Carvalho \& Chambel, 2014; Chan et al., 2016; Nicklin \& McNall, 2013). Comparativamente ao impacto do EFT no bemestar subjetivo, o impacto do ETF na saúde dos indivíduos tem sido menos estudado. No entanto, algumas investigações salientam que as pessoas que reportam níveis mais elevados de ETF têm uma maior probabilidade de experienciarem níveis superiores de saúde física e mental (e.g., McNall et al., 2010; Stoddard \& Madsen, 2007) ou de terem uma perceção mais positiva da sua saúde (e.g., Carvalho \& Chambel, 2014). De acordo com o estudo de Van Steenbergen e Ellemers (2009), quando os colaboradores reportavam mais experiências de harmonização dos seus papéis de trabalho e de família, melhor eles se sentiam fisicamente. Já Russo (2015), no seu estudo, demonstrou que o ETF contribuía para melhorar a saúde percebida dos indivíduos através do aumento da sua persistência para alcançar os seus objetivos e da redução da vulnerabilidade ao stress. Mais recentemente, Sprung e Jex (2017) demonstraram que o ETF reportado pelo indivíduo se encontrava positivamente relacionado com a saúde psicológica do cônjuge e negativamente relacionado com os sintomas físicos também sentidos por este.

No presente estudo, a saúde dos participantes foi avaliada através dos sintomas somáticos que os indivíduos diziam experienciar diariamente. Estes sintomas são definidos como manifestações físicas com 
um grande componente psicológico, sendo muitas vezes "uma resposta física a stressores no trabalho" (Spector \& Jex, 1998, p. 358).

Assim, esperamos que quando os professores consideram que as suas experiências diárias na escola os ajudam a melhorar a qualidade da sua vida familiar nesse dia, isso esteja associado a uma menor intensidade de sintomas somáticos reportados nesse dia. Desta forma, colocámos a seguinte hipótese:

Hipótese 1: 0 enriquecimento trabalho-família diário está negativamente associado à intensidade dos sintomas somáticos sentidos diariamente.

A Teoria da Conservação dos Recursos (TCR, Hobfoll, 1989, 2002) defende que a aquisição de recursos está associada a benefícios para a saúde do indivíduo. De acordo com esta teoria, aqueles que possuem um "reservatório mais sólido de recursos têm maiores probabilidades de promoverem e manterem o seu bem-estar e a sua saúde" (Hobfoll, 2002, p. 311). Isto acontece porque "as pessoas que têm recursos têm menores probabilidades de encontrarem circunstâncias stressantes que afetem negativamente o seu bem-estar psicológico e físico" (Hobfoll, 2002, pp. 317-318). Por outro lado, a existência de recursos, não só pode gerar novos recursos, como pode simplificar o desenvolvimento e a utilização de recursos já existentes, o que foi designado por "caravana de recursos" (Hobfoll, 2002, p. 318).

Segundo a Teoria da Conservação dos Recursos, os recursos são aquelas entidades que são centralmente valorizadas per se, podendo ser contextuais ou pessoais. Um recurso contextual é externo ao indivíduo, residindo no contexto social onde ele se encontra. No caso dos professores, poderão ser exemplos de recursos contextuais, o suporte social oferecido por um colega no trabalho, o feedback recebido por parte de um aluno, ou as oportunidades de desenvolvimento e de aprendizagem associadas ao desempenho desta profissão. Um recurso pessoal pode ser físico (como o vigor sentido), cognitivo (conhecimento, capacidade, experiência, capacidade de concentração), instrumental ou referente ao capital (dinheiro, tempo disponível), ou psicológico (resiliência, otimismo, auto-eficácia, etc.).

De acordo com a TCR, o processo de aquisição de recursos assenta na maximização dos recursos já existentes e na procura de novos, com consequente acumulação destes. À medida que se vão ganhando recursos (contextuais ou pessoais) é criada uma espiral de ganhos (Hobfoll, 1989, 2002; Van Steenbergen, Kluwer, \& Karney, 2014) associada a um incremento de emoções positivas. Em consonância, Ten Brummelhuis e Bakker (2012) afirmam que os recursos contextuais são um ponto de partida para os processos de enriquecimento do trabalho para a família, estando associados a uma espiral de ganhos assente na expansão da panóplia de recursos à disposição do indivíduo e no desenvolvimento de recursos pessoais.

Assim, da mesma forma que "as experiências de trabalho podem ser veículos de fortalecimento dos recursos psicológicos dos indivíduos” (Russo, 2015, p. 2489) potenciando o seu papel noutros domínios e conduzindo nomeadamente à experiência de EFT, faz sentido esperar que o EFT amplifique os recursos pessoais do indivíduo numa lógica de espiral de ganhos. Por exemplo, Russo (2015) verificou que níveis elevados de ETF estão associados a uma maior resiliência ao stress e a uma maior persistência nos objetivos. Deste modo, aqui pressupomos que o ETF, que tem por base a utilização dos recursos adquiridos em contexto organizacional no contexto familiar, pode despoletar uma espiral de ganhos gerando nomeadamente recursos pessoais como a autoeficácia, e que esses recursos psicológicos gerados podem explicar a relação entre o ETF sentido e os níveis mais elevados de saúde.

\section{Enriquecimento Trabalho-família e Autoeficácia}

A autoeficácia diz respeito à crença de que o indivíduo tem na sua "capacidade de organizar e executar os cursos de ação necessários para alcançar determinados tipos de desempenho" (Bandura, 1986, p. 391) com sucesso. Esta tem sido conceptualizada como traço ou como estado (Gardner \& Pierce, 1998). Quando nos referimos a traços, falamos sobretudo na autoeficácia associada à personalidade do indivíduo (Judge, Jackson, Shaw, Scott, \& Rich, 2007). Por outro lado, Bandura (2012) refere que "as pessoas se baseiam em parte nos seus estados físicos e emocionais para avaliar a sua autoeficácia” (p.13). As crenças que os indivíduos têm acerca das suas capacidades variam em função dos diferentes domínios de atividades e das condições situacionais em que se encontram, mais do que se manifestarem de forma uniforme nas diferentes tarefas e contextos à semelhança de um traço geral (Bandura, 2012). Em conformidade, a autoeficácia tem vindo a ser equacionada enquanto recurso pessoal que pode variar em função da situação específica vivida pela pessoa (Ten Brummelhuis \& Bakker, 2012; Russo, 2015). Por isso, neste estudo analisámos as variações intra-individuais da autoeficácia percebida e não a perceção de autoeficácia enquanto traço. Nomeadamente, explorámos de que forma as variações diárias do ETF sentido estão associadas a variações diárias na perceção de autoeficácia dos professores. E, por sua vez, de que forma estas mudanças quotidianas estão associadas a alterações diárias na intensidade dos sintomas somáticos reportados. 
Chan e colegas (2016) observaram que indivíduos que experienciam níveis mais elevados de ETF reportam níveis superiores de auto-eficácia específica à regulação da interface trabalho-família, um ano depois. 0 nosso estudo vem expandir o pouco que se sabe sobre a relação entre o ETF e a auto-eficácia ao centrar-se nas variações diárias e também ao analisar o impacto do EFT para a auto-eficácia na resolução de outro tipo de problemas, que não a articulação entre o trabalho e a família.

Assim, tendo em conta a TCR, espera-se que o ETF sentido diariamente gere uma espiral de ganhos podendo estar associado a uma perceção mais elevada de autoeficácia nesses dias: nos dias em que o indivíduo considera que foi capaz de transferir recursos do domínio do trabalho para o domínio familiar, isso estará associado a uma crença mais elevada nas suas capacidades para resolver situações problemáticas de uma forma geral. Desta forma, colocámos a seguinte hipótese:

Hipótese 2: o enriquecimento trabalho-família diário está positivamente associado à perceção de autoeficácia diária.

\section{Autoeficácia e saúde}

De acordo com Bandura (2012), as crenças de autoeficácia têm impacto na qualidade do funcionamento humano através de processos cognitivos, motivacionais, afetivos e decisionais. Já segundo Hobfoll (2002), as pessoas com níveis mais elevados de autoeficácia poderão ser mais capazes de selecionar e até implementar os seus outros recursos de forma a melhor lidarem com as exigências stressantes. De facto, a autoeficácia parece influenciar as capacidades de coping dos indivíduos (Bandura, 1988), o que "afeta a qualidade da sua vida emocional e a sua vulnerabilidade ao stress e à depressão" (Bandura, 2012, p.13). Por isso, podemos pensar que quanto maior for a autoeficácia percebida, menor será a tendência de um indivíduo para somatizar fatores de stress.

Alguns estudos têm vindo a demonstrar que a autoeficácia tem uma relação positiva, quer com o bem-estar psicológico (Bandura, 2006), quer com a saúde, bem como com alguns comportamentos de saúde dos indivíduos (e.g., Holden, 1992; Strecher, De Vellis, Becker, \& Rosenstock, 1986). Já Schwarzer e Hallum (2008), num estudo com professores, verificaram que níveis mais baixos de autoeficácia estavam associados a mais experiências de emoções negativas e distress, o que consequentemente levava ao burnout destes professores. Flett, Panico e Hewitt (2011) mostraram num estudo que analisava diferentes relações entre o perfecionismo, os comportamentos de tipo A, a autoeficácia, a depressão e os sintomas somáticos em adolescentes, que baixos níveis de autoeficácia se relacionavam significativamente com a depressão. Os autores verificaram ainda que havia uma relação negativa e significativa entre a autoeficácia e os sintomas somáticos reportados, indicando assim que os adolescentes com menor autoeficácia referiam sentir mais problemas de saúde. Assim, em consonância com a literatura anterior, esperamos que nos dias em que os professores sentem ter mais capacidades para lidarem com as situações problemáticas, relatem ter menos sintomas somáticos.

Hipótese 3: A perceção de autoeficácia diária está negativamente associada à intensidade dos sintomas somáticos sentidos diariamente.

\section{O papel mediador da autoeficácia percebida na relação entre o ETF e a saúde}

Os efeitos diretos que o ETF tem na saúde física e mental dos indivíduos (e.g., McNall et al., 2010) têm sido documentados, mas menos é sabido acerca dos mecanismos subjacentes a estas relações; ou seja, sobre quais poderão ser os processos envolvidos. Usando a Teoria da Conservação dos Recursos como referência, podemos esperar que o facto de o indivíduo sentir que os recursos gerados no trabalho podem melhorar a qualidade da sua vida familiar (ETF) pode conduzir a uma espiral ascendente de ganhos, nomeadamente em termos de recursos pessoais. De facto, alguns autores sugerem que o ETF contribui para o desenvolvimento, mobilização e acumulação de recursos físicos e psicológicos, os quais favorecem uma melhoria da saúde do indivíduo (e.g., Russo, 2015; Ten Brummelhuis \& Bakker, 2012). Assim, será de esperar que nos dias em que o indivíduo considera que conseguiu transferir recursos do trabalho para o domínio familiar e que isso lhe permitiu ser melhor membro da sua família, sinta também que teve maiores capacidades para resolver situações problemáticas (perceção de autoeficácia). Ainda de acordo com esta teoria, o aumento diário de recursos pessoais, (neste caso, a perceção de autoeficácia) pode ser um mecanismo central para a explicação da variação diária na saúde do indivíduo (cf. Hobfoll, 2002), nomeadamente no que respeita à intensidade dos sintomas somáticos reportados. De facto, Ten Brummelhuis e Bakker (2012) argumentam que a autoeficácia é um recurso psicológico chave que pode, não só ajudar os indivíduos a melhor lidarem com situações stressantes, como também a melhor otimizarem os outros recursos a que podem ter acesso. Por isso, faz sentido esperar que o aumento da autoeficácia diária esteja associado a níveis mais baixos de stress e, consequentemente, a níveis mais baixos de sintomas somáticos reportados. 
Neste trabalho testámos então, de forma empírica, o papel das variações diárias de autoeficácia para a explicação do processo através do qual o ETF sentido diariamente poderá afetar a saúde diária dos indivíduos, nomeadamente a intensidade dos sintomas somáticos sentidos. Colocámos por isso a seguinte hipótese:

Hipótese 4: 0 efeito do enriquecimento trabalho-família sentido diariamente na intensidade dos sintomas somáticos diários é mediado pelas variações diárias na autoeficácia percebida diariamente.

\section{MÉTODO}

Realizámos um estudo de tipo diário. A utilização deste tipo de estudo tem vindo a aumentar no âmbito da investigação sobre a interface trabalho-família (Butler, Grzywacz, Bass, \& Linney, 2005), visto que permite capturar eventos, disposições ou interações perto da altura em que estas ocorreram (Iida, Shrout, Laurenceau, \& Bolger, 2012). 0 estudo diário permite analisar as flutuações diárias (Ohly, Sonnentag, Niessem, \& Zapf, 2010) da forma como o trabalho interfere positivamente com a dimensão familiar e as suas consequências ao nível diário da saúde do indivíduo. Permite ainda estudar a autoeficácia, tendo em atenção, não a sua dimensão de traço, mas sim a sua dimensão de estado passível de ser influenciada por fatores contingenciais, como sejam, por exemplo, a experiência ETF sentida pelo indivíduo nesse dia.

\section{Participantes}

Recolhemos dados de professores de um agrupamento de escolas da região Oeste do país através de uma amostra de conveniência. Os participantes foram todos recrutados presencialmente. Dos 70 questionários distribuídos, foram entregues 66 válidos (94.2\% de taxa de resposta). Apenas 1 participante não respondeu a um dos oito momentos de medição. Assim, a amostra total contou com 66 professores, sendo $87.9 \%$ do sexo feminino. As idades estavam compreendidas entre os 35 e os 61 anos $(M=48.1, D P=6.68)$. 0 nível de habilitações dos participantes mais frequente era o de "Licenciatura" (72.7\%). A maior parte dos participantes lecionava o ensino secundário (51.5\%). Cerca de 45.5\% tinham uma carga letiva semanal de 22 horas ou mais e $65.2 \%$ detinham um contrato por tempo indeterminado. Quanto à sua situação familiar, 83.3\% dos participantes tinham filhos, sendo que a maior parte dos participantes tinha filhos com 20 (9.1\%) e 22 anos de idade (9.1\%). Cerca de 65.2\% viviam com o cônjuge/companheiro e os filhos.

\section{Procedimento}

No momento da recolha de dados foram garantidos o anonimato e a confidencialidade aos participantes das informações recolhidas, sendo foi ainda clarificado o principal objetivo do estudo. Foi pedido aos participantes que preenchessem um dia antes um questionário inicial que incluía informação sócio demográfica: sexo, idade, número de filhos, composição do seu núcleo familiar, antiguidade, carga letiva semanal, vínculo laboral, número de turmas, acumulação da atividade de professor com alguma outra responsabilidade e existência de alguma outra atividade remunerada. Após o preenchimento desse questionário foi solicitado aos participantes que preenchessem questionários diariamente durante 8 dias de trabalho consecutivos. Tanto a perceção de enriquecimento trabalho-família diária, como a autoeficácia sentida e a saúde (através da intensidade dos sintomas somáticos sentidos) foram medidas na manhã seguinte ao dia de trabalho, sendo os itens referentes ao que se passou no dia anterior. Cada participante recebia um pequeno caderno para este efeito. Uma vez que a utilização destes cadernos podia fazer com que houvesse algum incumprimento com o protocolo do estudo, fomos muito claras nas instruções dadas aos participantes, sublinhando a importância do seu preenchimento na altura do dia devida. Para além disso, incluímos nos cadernos um espaço para que os participantes registassem a que horas tinham preenchido os questionários.

\section{Instrumentos}

Dado que os participantes completavam questionários ao longo de 8 dias de trabalho, foi importante manter as escalas o mais curtas possível. Assim, utilizámos conjuntos reduzidos de itens de escalas validadas previamente em outros estudos diários.

Enriquecimento trabalho-família. 0 nível diário de enriquecimento trabalho-família foi operacionalizado através de 3 itens adaptados da escala curta de ETF de Kacmar, Crawford, Carlson, Ferguson e Whitten (2014). Foi utilizada uma escala de resposta do tipo Likert, de 7 pontos, em que o 1 correspondia a discordo totalmente e o 7 a concordo totalmente. 0 ETF diário foi medido de manhã e todos os itens foram ajustados de forma a fazerem referência ao dia anterior. Um exemplo de item foi: "Ontem o meu trabalho fez-me sentir feliz e isso ajudou-me a ser um melhor membro da minha família." 0 alfa de Cronbach das 8 ocasiões medidas foi de .85 . 
Autoeficácia. A autoeficácia geral foi medida através de 2 itens adaptados da escala de autoeficácia de Schwarzer e Jerusalem (1995). As respostas foram dadas numa escala do tipo Likert, de 7 pontos, em que o 1 correspondia a discordo totalmente e o 7 a concordo totalmente. 0 nível diário de autoeficácia foi medido de manhã e os itens foram ajustados de forma a fazerem referência ao dia anterior. Um exemplo de item foi: "Ontem senti confiança para poder lidar de forma eficaz com eventos inesperados". 0 coeficiente de Spearman Brown $\left(r_{S B}\right)$ médio das 8 ocasiões de medida foi de .70.

Sintomas somáticos. A saúde diária foi avaliada através dos sintomas somáticos que foram medidos com 10 itens adaptados da escala "Physical Symptoms Inventory" (PSI), de Spector e Jex (1998) com uma escala de intensidade de 7 pontos, em que o 1 correspondia a nunca e o 7 a todo o dia. 0 nível diário de sintomas somáticos foi medido de manhã e os itens foram ajustados de forma a fazerem referência ao dia anterior. Um exemplo de item foi: "tendo em conta o dia de ontem, indique com que intensidade sentiu náuseas/dores de estômago". 0 nível de Cronbach médio das 8 ocasiões de medida foi de .78.

Variáveis de controlo. 0 sexo, a idade e a carga letiva foram usadas como variáveis de controlo. A variável sexo ( 0 = masculino, 1 = feminino $)$ foi controlada, visto que alguns estudos sugerem que homens e mulheres têm experiências diferentes a nível do trabalho e da família (Barnett \& Hyde, 2001). As horas de trabalho são tipicamente um dos fatores de maior pressão para os professores (Yu, Wang, Zhai, Dai, \& Yang, 2015) e tendo em conta que vários autores (e.g., Gutek, Searle, \& Klepa, 1991; Marshall \& Barnett, 1993) referem que quando os indivíduos trabalham mais horas, maior é a probabilidade de o trabalho interferir com o bem-estar no seu seio familiar, optámos por controlar a carga letiva semanal destes profissionais.

\section{Estratégias de análise}

Após o processo de recolha foram inseridas as respostas aos questionários numa base de dados em SPSS. Uma vez que os nossos dados estão hierarquicamente estruturados estando os dias (Nível 1, $N=528$ observações) encaixados (i.e., nested) em pessoas (Nível 2, $N=66$ participantes), conduzimos uma análise multinível usand o o programa MLWIN (Rasbash, Browne, Healy, Cameron, \& Charlton, 2002). Os preditores de nível 2 (nomeadamente as variáveis de controlo) foram centrados usando a média de grupo (i.e., grand mean) e os preditores de nível diário foram centrados utilizando a média de cada pessoa. Assim, analisámos se níveis altos ou baixos de ETF sentido num dia, comparados com o nível de ETF diário sentido em média por essa pessoa, estava associado a níveis mais elevados de autoeficácia sentida e a níveis mais baixos de sintomas somáticos. Utilizámos a exclusão completa de casos (i.e., list-wise deletion) para gerir as não respostas (i.e., missings) em todas as nossas análises.

\section{RESULTADOS}

\section{Análises preliminares}

A Tabela 1 apresenta as médias $(M)$, desvios-padrão $(D P)$ e correlações para todas as variáveis do estudo. Foi calculada a média das medidas repetidas nos 8 dias das variáveis de nível diário (i.e., day level) para analisar a correlação entre essas médias e a variável "carga letiva” medida a um nível individual (i.e., individual level). Como pode ser observado na tabela 1, o padrão de correlações entre as variáveis foi no sentido esperado.

Tabela 1. Médias, desvios-padrão e correlações ( $\mathrm{N}=66$ indivíduos, $\mathrm{N}=528$ observações)

\begin{tabular}{|c|c|c|c|c|c|c|c|}
\hline Variável & $M(D P)$ & 1 & 2 & 3 & 4 & 5 & 6 \\
\hline 1. ETF & $4.4(1.1)$ & - & & & & & \\
\hline 2. Autoeficácia & $5.6(0.9)$ & $.36^{* * *}$ & - & & & & \\
\hline 3. Sintomas somáticos & $1.6(0.5)$ & $-.17^{* * *}$ & $-.21 * *$ & - & & & \\
\hline 4. Carga letiva & $5.1(1.8)$ & $.17^{* * *}$ & .001 & $.11 *$ & - & & \\
\hline 5. Sexo & $-(-)$ & $\ldots 17^{* * *}$ & -.02 & .03 & $.14^{* *}$ & - & \\
\hline 6. Idade & $48(0.7)$ & $-.11^{* *}$ & .02 & .07 & $-.33^{* * *}$ & .06 & - \\
\hline
\end{tabular}

Nota. ETF = enriquecimento trabalho-família; ${ }^{*} p<.05 ;{ }^{* *} p<.01,{ }^{* * *} \mathrm{p}<.001$

O ETF diário estava negativamente correlacionado com os sintomas somáticos $(r=-.17, p<.001) \mathrm{e}$ positivamente correlacionado com a autoeficácia percebida $(r=.36, p<.001)$. A autoeficácia percebida estava negativamente correlacionada com os sintomas somáticos $(r=-.21, p<.001)$. Quanto às variáveis de controlo, a carga letiva estava positivamente associada à intensidade dos sintomas somáticos 
reportados $(r=.11, p<.001)$; o sexo e a idade não estavam significativamente associados aos sintomas somáticos reportados diariamente. Como tal, por razões de parcimónia, só foi incluído no modelo de regressão a carga letiva como variável de controlo. De forma a compreender se a variável dos sintomas somáticos flutuava a nível diário, seguimos as recomendações de Bliese (2012) e testámos um modelo de médias incondicionais (i.e., unconditional means model) para calcular os valores das correlações intraclasse (ICC) e para testar se a variância do intercepto era significativamente diferente de zero. Os resultados mostram que o ICC1 para os sintomas somáticos era de .34, o que indica uma variância de 34\% nos sintomas somáticos reportados de dia para dia. No que diz respeito ao ETF, verificou-se uma variância de $43 \%$ no ETF reportado diariamente (ICC1 $1_{\mathrm{ETF}}=.43$ ). Ao evidenciarem que uma porção importante de variância dos sintomas e do ETF pode ser explicada pelas flutuações intra-individuais (i.e., within person) ao longo dos 8 dias do estudo, estes resultados indicam então a necessidade de utilizar um modelo multinível de análise.

\section{Teste das hipóteses}

Os resultados obtidos com cada um dos modelos encaixados (i.e., nested models) de efeitos variáveis usados para testar os efeitos de mediação apresentados na tabela 2 mostram as estimativas não estandardizadas, os erros-padrão e os valores de $t$ para todos os preditores. Os modelos multinível testados foram de efeitos aleatórios.

No modelo nulo, ou modelo de base (i.e., baseline model) incluímos o intercepto como único preditor. Este modelo contém apenas o intercepto e os erros (i.e., error terms) correspondentes (Hox \& Roberts, 2011). No Modelo 1, incluímos o ETF e a carga letiva (variável de controlo de nível pessoal) para testar se o ETF estava relacionado com a intensidade dos sintomas reportados, controlando a carga letiva do professor (H1) antes de introduzirmos a autoeficácia percebida (Modelo 2) de forma a testar se esta variável potencialmente mediadora estava relacionada com a intensidade dos sintomas somáticos reportados (H3). Comparámos o ajustamento destes modelos, calculando a diferença entre o rácio de verosimilhança (i.e., likelihood ratio) de cada modelo com o rácio de verosimilhança do modelo anterior. Esta diferença tem uma distribuição Qui-quadrado (sendo os graus de liberdade o número de variáveis adicionadas a cada modelo). 0 Modelo 2 evidenciou um ajustamento melhor do que o Modelo 1 (diferença de $-2 \times \log =13.34, g l=1, p<.001$ ), e do que o Modelo nulo (diferença de $-2 \times \log =67.19, g l=3, p<.001$ ).

Os resultados da análise multinível suportam a nossa primeira hipótese (H1), que pressupunha que o ETF diário estava negativamente relacionado com os sintomas somáticos reportados. De facto, o ETF diário estava negativamente associado à intensidade dos sintomas somáticos reportados diariamente quando controlávamos a carga letiva dos participantes $(\gamma=-.10, t=4.75, E P=.02, p<.001)$. Posteriormente, examinámos a relação entre o ETF diário e a autoeficácia percebida. Os resultados suportam a segunda hipótese (H2), pois o ETF diário estava positivamente associado à autoeficácia percebida reportada diariamente $(\gamma=.38, t=8.17, E P=.05, p<.001)$. De seguida, analisámos a relação entre a autoeficácia e os sintomas somáticos. No que diz respeito à nossa terceira hipótese (H3), os resultados evidenciam que a autoeficácia percebida reportada diariamente está negativamente associada aos sintomas diários reportados, apoiando a $\mathrm{H} 3(\gamma=-.09, t=3.67, E P=.02, p<.001)$.

Finalmente, testámos os efeitos indiretos do ETF diário nos sintomas somáticos reportados diariamente através da perceção da autoeficácia controlando a carga letiva dos professores (H4). 0 efeito do ETF diário nos sintomas reportados (Modelo 1) diminuiu em magnitude quando se introduziu na equação de regressão a autoeficácia percebida reportada diariamente (Modelo 2), mantendo-se, porém, estatisticamente significativo $(\gamma=-.06, t=2.82, E P=.02, p<.01)$.

Os resultados evidenciaram, portanto, a existência de uma mediação parcial (Mathieu \& Taylor, 2006), sendo, de acordo com Sobel (1982), o efeito indireto do ETF nos sintomas, através da autoeficácia, estatisticamente significativo (Sobel: $z=3.34, p<.001$ ). A hipótese 4 foi então parcialmente suportada pelos dados. 
Tabela 2. Estatísticas multinível para os modelos prevendo os sintomas somáticos dos professores $(\mathrm{N}=66$ professores, $\mathrm{N}=528$ observações)

\begin{tabular}{|c|c|c|c|c|c|c|c|c|c|}
\hline \multirow{2}{*}{ Variável } & \multicolumn{3}{|c|}{ Modelo nulo } & \multicolumn{3}{|c|}{ Modelo 1} & \multicolumn{3}{|c|}{ Modelo 2} \\
\hline & Est. & EP & $t$ & Est. & EP & $t$ & Est. & EP & $t$ \\
\hline Intercepto & 1.63 & .05 & $31.33^{* * *}$ & 1.63 & .05 & $31.80^{* *}$ & 1.62 & .05 & $31.82^{* * *}$ \\
\hline Carga letiva $^{a}$ & & & & .04 & .04 & .98 & .04 & .04 & 1.00 \\
\hline ETFb & & & & -.10 & .02 & $4.75^{* * *}$ & -.06 & .02 & $2.82^{* *}$ \\
\hline Autoeficáciab & & & & & & & -.09 & .02 & $3.67^{* * *}$ \\
\hline$-2 \times \log (\mathrm{lh})$ & & 309.21 & & & 255.35 & & & 242.02 & \\
\hline Diferença de $-2 \times \log$ & & & & & 58.86 & & & 13.34 & \\
\hline Graus de liberdade & & & & & 2 & & & 1 & \\
\hline $\begin{array}{l}\text { Variância do } \\
\text { intercepto nível } 1 \text { (EP) }\end{array}$ & & $.08(.01)$ & & & $.07(.01)$ & & & $.07(.01)$ & \\
\hline $\begin{array}{l}\text { Variância do } \\
\text { intercepto nível } 2 \text { (EP) }\end{array}$ & & $.17(.03)$ & & & $.16(.03)$ & & & $.16(.03)$ & \\
\hline
\end{tabular}

Notas: $\mathrm{ETF}=$ enriquecimento trabalho-família; Est. = estimativa; EP = erro padrão; ${ }^{* * *} p<.001,{ }^{* *} p<.01 ;{ }^{a}$ preditor ao nível individual; b preditores ao nível do dia.

\section{DISCUSSÃO}

O presente estudo tinha como principal objetivo analisar o impacto das variações diárias do ETF na saúde do indivíduo, nomeadamente nos sintomas somáticos que este reportava diariamente. Pretendíamos ainda averiguar o papel que a perceção de autoeficácia tinha para a explicação desta relação. Os resultados das análises efetuadas suportam as hipóteses colocadas, ao demonstrarem que o ETF diário estava negativamente relacionado com os sintomas somáticos reportados, quando controlada a carga letiva dos participantes (H1) e positivamente com a autoeficácia (H2). Esta, por sua vez, relaciona-se de forma negativa com os sintomas somáticos reportados diariamente pelos participantes (H3). 0 efeito do EFT nos sintomas somáticos revelou ser parcialmente mediado pela perceção de autoeficácia geral do indivíduo (H4). Olhando para os nossos resultados, verificamos então que, nos dias em que o indivíduo se sente mais capaz de transferir a sua experiência do trabalho para a sua vida familiar, ele sente-se mais capaz de alcançar o que pretende de uma forma bem-sucedida (níveis mais elevados de autoeficácia diária), o que apoia o estudo de Chan e colegas (2016), que evidenciou uma relação positiva entre o ETF sentido e a autoeficácia percebida pelo indivíduo no que respeita à articulação entre o trabalho e a vida familiar. Por sua vez, o presente estudo demonstrou que a perceção acrescida de autoeficácia do indivíduo está associada à manifestação de um menor número de sintomas somáticos, o que nos leva a concluir que o ETF sentido diariamente está associado a níveis mais elevados de saúde. É de salientar ainda que a relação entre o ETF diário e os sintomas somáticos é em parte explicada pelo acréscimo da autoeficácia que resulta do ETF sentido.

No seguimento do trabalho de vários autores que recorreram à Teoria da Conservação dos Recursos para um melhor entendimento dos processos de enriquecimento que acontecem entre o universo do trabalho e o universo da família, o nosso estudo permite expandir e alargar os conhecimentos teóricos e empíricos acerca das espirais de ganhos e de como o aumento dos recursos pessoais possibilitados pelo EFT pode explicar a relação desta sinergia trabalho-família com a auto-eficácia percebida e com a intensidade dos sintomas somáticos reportados. Particularmente, os nossos resultados apoiam a ideia de que nos dias em que o indivíduo sente que possui mais recursos, ele faz uma avaliação mais positiva da sua capacidade de resolver situações problemáticas e da sua saúde, reportando um menor número de sintomas somáticos. Estes resultados vão ao encontro do proposto por Greenhaus e Powell (2006), que referiam que o gerar de recursos que o processo de enriquecimento desencadeia parece favorecer a saúde dos indivíduos (McNall et al., 2010), mas também da TCR, de acordo com a qual a acumulação de recursos não só gera novos recursos como pode, por isso, beneficiar o bem-estar e a saúde dos indivíduos. .

Este estudo vem contribuir para ampliar a literatura acerca do enriquecimento trabalho-família. Mais especificamente, o presente estudo permitiu aprofundar o conhecimento sobre o impacto do ETF na saúde do indivíduo e o mecanismo que, em parte, explica este efeito positivo: o aumento da perceção de autoeficácia enquanto recurso pessoal. Também o facto de termos realizado um estudo diário é um importante contributo, pois conseguimos compreender e analisar as flutuações diárias do ETF, da autoeficácia e dos sintomas somáticos e a relação entre estas variáveis (Ohly et al., 2010). A amostra do nosso estudo era composta por professores, o que permitiu enriquecer a literatura acerca da interface trabalho-família neste tipo de profissionais. 


\section{Limitações e Estudos Futuros}

Não obstante os contributos já enunciados, esta pesquisa apresenta algumas limitações. Primeiro, todas as nossas medidas foram autoreportadas, o que pode ter levado a um aumento da variância comum entre as variáveis (Podsakoff, Mackenzie, Lee, \& Podsakoff, 2003). Segundo, o facto de termos realizado um estudo diário faz com que tenhamos apenas dados correlacionais, o que, apesar da temporalidade na recolha da informação, não nos permite estabelecer inferências de causalidade. De facto, não podemos excluir uma possível circularidade das relações causais, isto porque um indivíduo com menos sintomas pode avaliar a sua capacidade de lidar com situações problemáticas de forma mais positiva (maior autoeficácia percebida). Por sua vez, um indivíduo com autoavaliações mais positivas poderá ter tendência a sobreavaliar a sua capacidade de transpor os recursos obtidos no trabalho para a sua vida familiar. Seria, por isso, importante realizar um estudo diário que medisse as variáveis em momentos diferentes do tempo. Terceiro, no nosso estudo diário, os participantes relataram a perceção das variáveis em estudo referentes ao dia anterior. Embora esta medida possa ser vantajosa por ser uma avaliação que engloba todo o dia anterior, não podemos excluir a hipótese de poder haver algum enviesamento na recordação (por exemplo, dependendo da qualidade do sono e de um possível efeito de recuperação, ou de outras variáveis externas presentes durante a noite). No entanto, o hiato temporal é tão curto que julgamos que esse potencial efeito de recuperação poderá não afetar significativamente os nossos resultados, não levando necessariamente a uma reconstrução da experiência do dia anterior. Uma outra limitação reside no facto de os estudos diários implicarem o uso de medidas repetidas intensivas. Esta repetição pode provocar cansaço e desmotivação na forma como os participantes respondem ao longo do tempo. A avaliação repetida das variáveis ao longo dos dias pode conduzir ainda a uma maior consciencialização de algumas cognições e comportamentos, podendo levar a uma possível modificação do comportamento (Tennen, Affleck, Armeli, \& Carney, 2000). Porém, nada indica que tenha havido mudanças comportamentais na forma como os participantes responderam ao longo do estudo devido ao eventual aumento da sua auto-monitorização. Por último, a nossa amostra era de conveniência, o que poderá ameaçar a possibilidade de generalização dos resultados a outras populações com características diferentes das características dos professores enquanto grupo profissional.

Assim, estudos futuros poderiam tentar recolher dados de diferentes fontes, por exemplo, utilizando o ETF do indivíduo, mas reportado pelo parceiro. Também poderia ser pertinente em estudos futuros analisar o papel de outros recursos pessoais, para além da autoeficácia, para a explicação da relação entre o ETF e a saúde dos indivíduos. Por exemplo, os recursos energéticos (Tavares \& Dias, 2014), outros recursos psicológicos (como a perceção de controlo ou o otimismo), recursos cognitivos (como a capacidade de concentração), ou recursos de capital (como o tempo disponível). Em vez de um estudo diário, também poderia ser interessante realizar um estudo longitudinal.

\section{Implicações Práticas}

Do ponto de vista mais prático, os nossos resultados apontam para a importância de que os responsáveis das organizações levem a cabo iniciativas que promovam o ETF, pois estas podem contribuir para que os seus colaboradores construam não só uma crença mais forte de autoeficácia, mas também para que manifestem um menor número de sintomas somáticos. Assim, incentivar o ETF pode trazer consequências positivas não só para o indivíduo, mas também para a sua organização e para a sua família a longo prazo (Hakanen, Peeters, \& Perhoniemi, 2011). Enriquecer o trabalho dos indivíduos, enriquecendo os seus recursos no trabalho pode ser fundamental (Van Steenbergen et al., 2014). Isto pode ser feito a título de exemplo, com mais oportunidades de desenvolvimento (Van Steenbergen et al., 2014), com o aumento da segurança do vínculo laboral (Carlson et al., 2011) ou intervindo ao nível do desenho do trabalho aumentando a variedade das tarefas (Carlson et al., 2011) ou a autonomia no trabalho (Siu et al., 2010). A promoção do apoio do supervisor (Siu et al., 2010) e o suporte da organização nos domínios da vida do indivíduo fora do trabalho (Kelly et al., 2008) poderão ser também fatores importantes para a promoção do ETF. Para além disso, o impacto positivo do ETF na autoeficácia enfatiza ainda a necessidade de as organizações considerarem o domínio familiar como facilitador das crenças de autoeficácia dos trabalhadores e do seu bem-estar (Chan et al., 2016).

Em suma, esta pesquisa sugere que o ETF tem efeitos benéficos para a saúde dos indivíduos em virtude dos recursos pessoais que potencia, nomeadamente a autoeficácia percebida. Este estudo contribui de forma importante para a literatura, sendo um dos primeiros estudos a testar empiricamente os mecanismos que explicam porque o ETF sentido num dia pode diminuir os sintomas somáticos experienciados pelo indivíduo nesse mesmo dia. 


\section{REFERÊNCIAS}

Aryee, S., Srinivas, E. S., \& Tan, H.H. (2005). Rhythms of life: Antecedents and outcomes of work-family balance in employed parents. Journal of Applied Psychology, 90 (1), 132-146.

Bandura, A. (1986). Social foundations of thought and action: A social cognitive theory. Prentice Hall.

Bandura, A. (1988). Organizational applications of social cognitive theory. Australian Journal of Management, 13 (2), 275-302.

Bandura, A. (2006). Adolescent development from an agentic perspective. In F. Pajares \& T. Urdan (Eds.), Self-efficacy beliefs of adolescentes (pp. 1-43). Information Age Publishing.

Bandura, A. (2012). On the functional properties of perceived self-efficacy revisited. Journal of Management, 38 (1), 9-44.

Barnett, R. C., \& Hyde, J. S. (2001). Women, men, work, and family: An expansionist theory. American Psychologist, 56 (10), 781-796.

$\begin{array}{lllllll}\text { Bliese, } & \text { P. } & \text { (2012). } & \text { Multilevel modeling } & \text { in } & \text { (2.4). }\end{array}$ http://cran.rproject.org/doc/contrib/Bliese_Multilevel.pdf

Butler, A. B., Grzywacz, J. G., Bass, B. L., \& Linney, K. D. (2005). Extending the demands-control model: A daily diary study of job characteristics, work-family conflict, and work-family facilitation. Journal of Occupational and Organizational Psychology, 78 (2), 155-169.

Carlson, D. S., Kacmar, M. K., Wayne, J. H., \& Grzywacz, J. G. (2006). Measuring the positive side of the workfamily interface: Development and validation of a work-family enrichment scale. Journal of Vocational Behavior, 68 (1), 131-164.

Carlson, D. S., Kacmar, M. K., Zivnuska, S., Ferguson, M. \& Whitten, D. (2011). Work-family enrichment and job performance: a constructive replication of affective events theory. Journal of Occupational Health Psychology, 16 (3), 297-312.

Carvalho, V. S., \& Chambel, M. J. (2014). Work-to-family enrichment and employees' well-being: High performance work system and job characteristics. Social Indicators Research, 119 (1), 373-387.

Carvalho, V. S., \& Chambel, M. J. (2018). Work-family conflict and enrichment mediates the relationship between job characteristics and well-being at work with Portuguese Marine Corps. Armed Forces \& Society, 44 (2), 301-321.

Chan, X. W., Kalliath, T., Brough, P., Siu, O. L., O'Driscoll, M. P., \& Timms, C. (2016). Work-family enrichment and satisfaction: The mediating role of self-efficacy and work-life balance. International Journal of Human Resource Management, 27 (15), 1755-1776.

Cho, E., \& Chen, T.-Y. (2018). The effects of work-family experiences on health among older workers. Psychology and Aging, 33 (7), 993-1006.

Cinamon, R. G. (2009). Role salience, social support, and work-family conflict among jewish and arab female teachers in Israel. Journal of Career Development, 36 (2), 139-158.

Daniel, S., \& Sonnentag, S. (2014). Work to non-work enrichment: The mediating roles of positive affect and positive work reflection. Work \& Stress, 28 (1), 49-66.

De Simone, S., Cicotto, G., \& Lampis, J. (2016). Occupational stress, job satisfaction and physical health in teachers. Revue Européenne de Psychologie Appliquée, 66 (2), 65-77.

Flett, G. L., Panico, T., \& Hewitt, P. L. (2011). Perfectionism, type A behavior, and self-efficacy in depression and health symptoms among adolescents. Current psychology, 30, 105-116.

Frone, M. R. (2003). Work-family balance. In. J. C Quick \& L. E Tetrick (Eds.), Handbook of occupational health psychology (pp. 143-162). American Psychological Association.

Gardner, D. G., \& Pierce, J. L. (1998). Self esteem and self-efficacy within the organizational context: An empirical examination. Group \& Organization Management, 23 (1), 48-70.

Greenhaus, J. H., \& Powell, G. N. (2006). When work and family are allies: A theory of work-family enrichment. Academy of management review, 31 (1), 72-92.

Gutek, B. A., Searle, S., \& Klepa, L. (1991). Rational versus gender role explanations for work-family conflict. Journal of Applied Psychology, 76 (4), 560-568.

Hakanen, J. J., Peeters, M. C. W., \& Perhoniemi, R. (2011). Enrichment processes and gain spirals at work and at home: A 3-year cross-lagged panel study. Journal of Ocupational and Organizational Psychology, 84 (1), 8-30.

Hobfoll, S. E. (1989). Conservation of resources: A new attempt at conceptualizing stress. American Psychologist, 44 (3), 513-524.

Hobfoll, S. E. (2002). Social and psychological resources and adaptation. Review of General Psychology, 6 (4), 307-324.

Holden, G. (1992). The relationship of self-efficacy appraisals to subsequent health related outcomes: A meta-analysis. Social work in health care 16 (1), 53-93.

Hox, J. J., \& Roberts, J. K. (2011). Handbook of advanced multilevel analysis. Routledge. 
Iida, M., Shrout, P. E., Laurenceau, J. P., \& Bolger, N. (2012). Using diary methods in psychological research. In. H. Cooper, P. M. Camic, D. L. Long, A. T. Panter, D. Rindskopf \& K. J. Sher (Eds.), APA handbook of research methods in psychology, Foundations, planning, measures, and psychometrics (pp. 277-305). American Psychological Association.

Judge, T. A., Jackson, C. L., Shaw, J. C., Scott, B. A., \& Rich, B. L. (2007). Self-efficacy and work-related performance: The integral role of individual differences. Journal of Applied Psychology, 92 (1), 107 127.

Kacmar, K. M., Crawford, W. S., Carlson, D. S., Ferguson, M., \& Whitten, D. G. (2014). A short and valid measure of work-family enrichment. Journal of Occupational Health Psychology. 19 (1), 32-45

Kelly, E. L., Kossek, E.E, Hammer, L.B., Durham, M., Bray, J., Chermack, K, Murphy, L.A. \& Kaskubar, D. (2008). Getting there from here: Research on the effects of work-family initiatives on work- family conflict and business outcomes. The Academy of Management Annals, 2 (1), 305-349.

Kidger, J., Brockman, R., Tilling, K., Campbell, R., Ford, T., Araya, R., King, M., \& Gunnell, D. (2016). Teachers' wellbeing and depressive symptoms, and associated risk factors: A large cross-sectional study in English secondary schools. Journal of affective disorders, 192, 76-82.

Liu, H., \& Cheng, F. M. (2015). The role of work-family role integration in a job demands-resources model among Chinese secondary school teachers. Asian Journal of Socual Psychology, 18 (4), 288-298.

Marks, S. R. (1977). Multiple roles and role strain: Some notes on human energy, time and commitment. American Sociological Review, 42 (6), 921-936.

Marshall, N. L., \& Barnett, R. C. (1993). Work-family strains and gains among two-earner couples. Journal of Community Psychology, 21 (1), 64-78.

Mathieu, J. E., \& Taylor, S. R. (2006). Clarifying conditions and decision points for meditational type inferences in organizational behaviour. Journal of Organizational Behavior, 27 (8), 1031-1056.

McNall, L. A., Nicklin, J. M., \& Masuda, A. D. (2010). A meta-analytic review of the consequences associated with work-family enrichment. Journal of Business and Psychology, 25 (3), 381-396.

McNall, L. A., Scott, L., \& Nicklin, J. M. (2015). Do positive affectivity and boundary preferences matter for work-family enrichment? A study of human service workers. Journal of Occupational Health Psychology, 20 (1), 93-104.

Nicklin, J. M., \& McNall, L. A. (2013). Work-family enrichment, support, and satisfaction: A test of mediation. The European Journal of Work and Organizational Psychology, 22 (1), 67-77.

Ohly, S., Sonnentag, S., Niessen, C., \& Zapf, D. (2010). Diary studies in organizational research: an introduction and some practical recommendations. Journal of Personnel Psychology, 9 (2), 79-93.

Padrão, I., Pinto, C., \& Santos-Rita, J. (2012). Bem-estar e estratégias de gestão das exigências em professores portugueses dos diferentes níveis de ensino. Actas do 12.ํㅡ Colóquio de Psicologia e Educação, 575-585.

Panayiotis, A., \& Kyrickides, L. (2013). A dynamic integrated approach to teacher professional development: Impact and sustainability of the effects on improving teacher behaviour and student outcomes. Teaching and Teacher Education, 29, 1-12.

Podsakoff, P. M., MacKenzie, S. M., Lee, J., \& Podsakoff, N. P. (2003). Common method variance in behavioral research: A critical review of the literature and recommended remedies. Journal of Applied Psychology, 88 (5), 879-903.

Rasbash, J., Browne, W., Healy, M., Cameron, B., \& Charlton, C. (2002). MLwiN (Version 1.10.006): Interactive software for multilevel analysis. Centre for Multilevel Modelling, Institute of Education, University of London.

Russo, M. (2015). Work-home enrichment and health: an analysis of the mediating role of persistence in goal striving and vulnerability to stress. The International Journal of Human Resource Management, 26 (19), 2486-2502.

Schwarzer, R., \& Hallum, S. (2008). Perceived teacher self-efficacy as a predictor of job stress and burnout: Mediation analyses. Applied Psychology: An International Review. Special Issue: Health and WellBeing, 57 (s1), 152-171.

Schwarzer, R., \& Jerusalem, M. (1995). Generalized Self-Efficacy scale. In. J. Weinman, S. Wright, \& M. Johnston (Eds). Measures in health psychology: A user's portfolio. Causal and control beliefs (pp. 3537). Nfer Nelson.

Sieber, S. D. (1974). Toward a theory of role accumulation. American Sociological Review, 39(4), 567-578.

Siu, O., Lu, J., Brough, P., Lu, C., Bakker, A.B., Kalliath, T., O’Driscoll, M., Phillips, D.R., Chen, W., Lo, D., Sit, C., \& Shi, K. (2010). Role resources and work-family enrichment: The role of work engagement. Journal of Vocational Behavior, 77 (3), 470-480.

Sobel, M. E. (1982). Asymptotic intervals for indirect effects in structural equations models. In S. Leinhart (Ed.), Sociological methodology (pp.290-312). Jossey-Bass. 
Spector, P. E., \& Jex, S. M. (1998). Development of four self-report measures of job stressors and strain: Interpersonal conflict at work scale, organizational constraints scale, quantitative workload inventory, and physical symptoms inventory. Journal of Occupational Health Psychology, 3 (4), 356367.

Sprung, J. M., \& Jex, S. M. (2017). All in the family: An examination of work-family enrichment and crossover among farm couples. Journal of Occupational Health Psychology, 22 (2), 218-224

Stoddard, M. \& Madsen, S. R. (2007). Toward an understanding of the link between work-family enrichment and individual health. Journal of Behavioral and Applied Management, 9 (1), 2-15.

Strecher, V. J., DeVellis, B. M., Becker, M. H., \& Rosenstock, I. M. (1986). The role of self-efficacy in achieving health behavior change. Health Education Quarterly, 13 (1), 73-92.

Tavares, S. M. \& Dias, A. (2014). Do conflito trabalho-família à (in)satisfação com a vida: Um caminho trilhado pela diminuição do vigor. In. M. L. Lima, S. Bernardes \& S. Marques (Eds). Psicologia Social da Saúde: Estudos, Programas e Instrumentos (pp. 89-105). Sílabo.

Ten Brummelhuis, L.L., \& Bakker, A.B. (2012). A resource perspective on the work-home interface: The work-home resources model. American Psychologist, 67 (7), 545-556.

Tennen, H., Affleck, G., Armeli, S., \& Carney, M. A. (2000). A daily process approach to coping: Linking theory, research, and practice. American Psychologist, 55 (6), 626-636.

Thoonen, E.E.J., Sleegers, P.L.C, Peetsma, T.T.D., \& Oort, F.J. (2011) Can teachers motivate students to learn?, Educational Studies, 37 (3), 345-360.

Van Steenbergen, E. F., \& Ellemers, N. (2009). Is managing the work-family interface worthwhile? Benefits for employee health and performance. Journal of Organizational. Behavior, 30 (5), 617-642.

Van Steenbergen, E. F., Kluwer, E. S., \& Karney, B. R. (2014). Work-family enrichment, work-family conflict and marital satisfaction: A dyadic analysis. Journal of Occupational and Health Psychology, 19 (2), 182-194.

Yu, X., Wang, P., Zhai, X., Dai, H., \& Yang, Q. (2015). The effect of work stress on job burnout among teachers: The mediating role of self-efficacy. Social Indicators Research, 122 (3), 701-708.

\begin{tabular}{lr}
\multicolumn{2}{c}{ Historial do artigo } \\
Recebido & $03 / 2019$ \\
Aceite & $07 / 2020$ \\
Publicado & $08 / 2020$
\end{tabular}

